\title{
On the Evolution of Approaches to the Space-Time Symmetry
}

\author{
Mikhail Petelin', Manfred Thumm² \\ ${ }^{1}$ Institute of Applied Physics, Russian Academy of Sciences, Nizhny Novgorod, Russia; ${ }^{2}$ Institute for Pulsed Power \\ and Microwave Technology, Karlsruhe Institute of Technology, Karlsruhe, Germany
}

Correspondence to: Mikhail Petelin, petelin@appl.sci-nnov.ru; Manfred Thumm, manfred.thumm@kit.edu Keywords: Space-Time Symmetry, Ecclesiastes, Aristotle, Proclus, Galileo, Michelson, Voigt, “Aether”, Lorentz Transformation, Theory of Relativity

Received: January 3, 2018 Accepted: March 4, 2018 Published: March 7, 2018

Copyright $\odot 2018$ by authors and Scientific Research Publishing Inc.

This work is licensed under the Creative Commons Attribution International License (CC BY 4.0).

http://creativecommons.org/licenses/by/4.0/

\section{(c) (1) Open Access}

\section{ABSTRACT}

The general space-time composition was analyzed starting in ancient times. During the previous three millenniums, to explain elementary physical phenomena, the world was twice admitted to be immersed into an "aether". Presently the "aether" is assumed to be asymptotically close to the vacuum. This approximation is included in the foundation of the special theory of relativity.

\section{ANCIENT SPACE-TIME SYMMETRY}

Primary opinions about the world harmony and symmetry were summarized by the Ecclesiastes (King Solomon, 990-931 BC) [1]: "Everything has its own time, and nothing is new under the Sun". A half millennium later, Aristotle (384-322 BC) turned prone to the "horror vacuum" and speculated that the nature is immersed into an "aether" [2]. Another millennium later, Proclus (412-485 AD-the last head of the Athens Academy) dismissed Aristotle's absolute reference frame for being not related to any physical phenomena [3].

\section{GALILEO'S REFERENCE FRAMES}

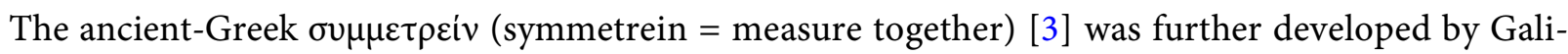
leo (1564-1642) [4,5] who used to illustrate the equivalence of all inertial reference frames with floating boats (Figure 1), where the times $\tau, \tau^{\prime}$ would be measured with beats of the man's heart and the coordinates $\xi, \xi^{\prime}$ would be measured with steps of the man's foot (the two sailors being assumed identical).

By imposing a common time-coordinate unit (conditionally equal to $M$ beats of heart $=N$ steps of foot $=1$ buck), the space-time homogeneity-isotropy $[1,3,5]$ might be met, if the times $\tau$, $\tau^{\prime}$ and coordinates $\xi, \xi^{\prime}$ were interrelated with modernized [6-8] symmetric equations as 


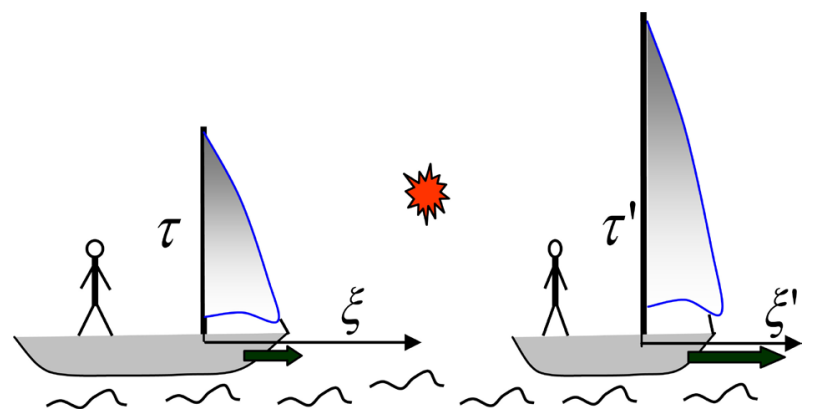

Figure 1. A Proclus-Galileo composition of inertial reference frames.

$$
\begin{array}{ll}
\xi=\gamma \xi^{\prime}+\kappa \tau^{\prime} & \xi^{\prime}=\gamma \xi-\kappa \tau \\
\tau=\kappa \xi^{\prime}+\gamma \tau^{\prime}, & \tau^{\prime}=-\kappa \xi+\gamma \tau
\end{array}
$$

$\gamma$ and $\kappa$ being constant coefficients. Inserting $\xi=0$ or $\xi^{\prime}=0$ into Equation (1) gives the mutual velocities

$$
\beta= \pm \kappa / \gamma
$$

of the reference frames.

If the coefficients $\gamma$ and $\kappa$ were arbitrary, the formula (2) would admit an excessive freedom for motion of material bodies. But fortunately, according to Proclus [3], the Aristotle's "horror vacuum" is withstood by the space-time symmetry itself. Indeed, mutual substitutions between the columns (1) give identities under the condition ${ }^{*}$ )

$$
\gamma^{2}-\kappa^{2}=1
$$

and the combination of (2) and (3) gives the relation

$$
\gamma=1 / \sqrt{1-\beta^{2}}
$$

imposing the absolute speed limit for the reference frames and thus for any material bodies

$$
|\beta| \leq 1
$$

*) Under the Minkowski change of variables [9], the left and right columns in (1) turn interrelated with rotation of relevant Descartes coordinates and, accordingly [8], the cyclic recurrence condition (3) turns equivalent to the theorem of Pythagoras. reproducing the $12^{\text {th }}$ theorem of Proclus [3]: "Ev

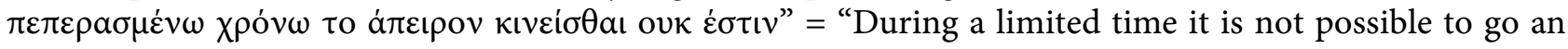
infinite distance" $\left.{ }^{\star}\right)$.

\section{RECENT UP AND DOWN OF THE "AETHER"}

However, in the second part of the $2^{\text {nd }}$ millennium $\mathrm{AD}$, the Ecclesiastes-Proclus-Galileo's concept of the space-time-symmetry $[1,3,5]$ underwent a new irruption by the Aristotle's "aether": experiments and theories by Grimaldi, Newton, Huygens, Young and Fresnel revealed that the light is a wave [4]; Maxwell postulated it being electromagnetic [10]; and that wave (by analogy with the sound and the sea waves) seemed [11] to need a special medium to propagate in.

The electromagnetic "aether" was anticipated to move relative to the Earth with a velocity commensurable to the velocity of the Sun relative to the Earth. To detect the "wind" caused with the motion of the "Luminiferous Aether", Michelson and Morley used an interferometer [12] situated on a massive rotatable stone plate floating in a mercury bath; but, contrary to expectations, the measurements did not admit the "aether" velocity that would exceed one tenth of the Earth-to-Sun velocity. The light seemed to propagate in all directions with a practically common velocity $\left(c=3 \times 10^{8} \mathrm{~m} / \mathrm{s}\right)$ [12]. The "paradox" was met by 
Voigt [13] who, following the Ecclesiastes approach [1], provided every reference frame with its own time $\left(t\right.$ or $\left.t^{2}\right)$ and coordinate $\left(x\right.$ or $\left.x^{2}\right)$ scales and described the wave propagation with equations (cited in the introduction to the book [9] of Pauli):

$$
\begin{gathered}
\frac{\partial^{2} \varphi}{\partial \xi^{2}}=\frac{\partial^{2} \varphi}{\partial \tau^{2}} \Leftrightarrow \frac{\partial^{2} \varphi}{\partial \xi^{\prime 2}}=\frac{\partial^{2} \varphi}{\partial \tau^{\prime 2}} \\
\xi=x, \quad \tau=c t, \quad \xi^{\prime}=x^{\prime}, \quad \tau^{\prime}=c t^{\prime} .
\end{gathered}
$$

$\varphi$ being the wave field potential.

The Voigt's symmetric hyperbolic Equation (6) were cyclic-recurrent under the coordinate-time transformation (1) and (3) which, combined with (7) was identical with the Lorentz transformation [9] $\left.{ }^{* *}\right)$.

\section{CONCLUSIONS}

The Aristotle's "aether" has been

- Converted by the special theory of relativity $[9,14]$ to a synonym of the vacuum-in agreement with the Ecclesiastes-Proclus-Galileo space-time symmetry $[1,3,5]$.

- Replaced by the gravitation field in generalized relativistic theories [9].

*) According to (1), if a body moves with a velocity $\beta_{b}^{\prime}$ relative to the $\xi^{\prime}, \tau^{\prime}$ frame, the body velocity relative to the $\xi, \tau$ frame is

$$
\beta_{b}=\frac{\beta_{b}^{\prime}+\beta}{1+\beta \beta_{b}^{\prime}}
$$

and if a body velocity is ultimate $\left(\beta_{b}^{\prime}=1\right)$ in one reference frame, it turns ultimate $\left(\beta_{b}=1\right)$ in any other reference frame as well [8].

${ }^{\star *}$ ) Lorentz in a footnote at page 198 of his book "Theory of Electrons" [15] pointed out that the Voigt's coordinate-time transformation [13] was an earlier version of his (Lorentz) transformation, but that he did not know about it at that time.

\section{REFERENCES}

1. Diderot, D. (1752) Ecclesiastes. Canon, 2, 601-604.

2. Chappell, V. (1973) Aristotle's Conception of Matter. Journal of Philosophy, 70, 679-696. https://doi.org/10.2307/2025076

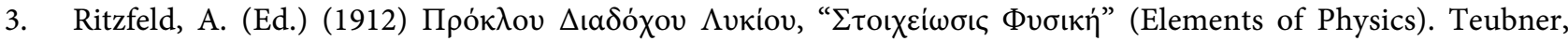
Leipzig. (Translation into German)

4. Gliozzi, M. (1965) Storia della Fisica (History of Physics). Torino, Italy.

5. Drake, S. (1978) Galileo at Work-His Scientific Biography. University of Chicago Press, Chicago, USA.

6. Feigenbaum, M.J. (2008) The Theory of Relativity-Galileo's Child. arXiv:0806.1234v1

7. Mermin, N.D. (1984) Relativity without Light. American Journal of Physics, 52, 119-124. https://doi.org/10.1119/1.13917

8. Petelin, M. (2015) The Universal Speed Limit as an Attribute of the Space-Time Symmetry. Journal of Basic and Applied Physics (JBAP), 4, 8-11.

9. Pauli, W. (1921) Theory of Relativity. Encyclopedia of Mathematical Sciences, 5-2.

10. Maxwell, J.C. (1873) A Treatise on Electricity and Magnetism. Clarendon Press, Oxford.

11. Schaffer, K.F. (1972) Nineteenth-Century Aether Theories. Pergamon Press, Oxford. 
12. Michelson, A.A. and Morley, E.W. (1887) On the Relative Motion of the Earth and the Luminiferous Ether. American Journal of Science, 34, 333-345. https://doi.org/10.2475/ajs.s3-34.203.333

13. Voigt, W. (1887) Ueber das Doppler'sche Princip (On Doppler's Principle). Nachrichten von der Königlichen Gesellschaft der Wissenschaften und der Georg-Augusts-Universität zu Göttingen, 8, 41-51.

14. Einstein, A. (1905) Zur Elektrodynamik bewegter Körper (On the Electrodynamics of Moving Bodies). Annalen der Physik, 17, 891-921. https://doi.org/10.1002/andp.19053221004

15. Lorentz, H.A. (1909) Theory of Electrons. B.G. Teubner, Leipzig. 